\title{
Electrocardiogram Derived Respiratory Rate Using a Wearable Armband
}

\author{
Jesús Lázaro*, Member, IEEE, Natasa Reljin, Member, IEEE, Raquel Bailón, Eduardo Gil, \\ Yeonsik Noh, Member, IEEE, Pablo Laguna, Fellow, IEEE, and Ki H. Chon, Senior Member, IEEE
}

\begin{abstract}
A method for deriving respiratory rate from an armband, which records three-channel electrocardiogram (ECG) using three pairs of dry (no hydrogel) electrodes, is presented. The armband device is especially convenient for long-term (monthsyears) monitoring because it does not use obstructive leads nor hydrogels/adhesives, which cause skin irritation even after few days. An ECG-derived respiration (EDR) based on respirationrelated modulation of QRS slopes and $R$-wave angle approach was used. Moreover, we modified the EDR algorithm to lower the computational cost. Respiratory rates were estimated with the armband-ECG and the reference plethysmography-based respiration signals from 15 subjects who underwent breathing experiment consisting of five stages of controlled breathing (at 0.1 , $0.2,0.3,0.4$, and $0.5 \mathrm{~Hz}$ ) and one stage of spontaneous breathing. The respiratory rates from the armband obtained a relative error with respect to the reference (respiratory rate estimated from the plethysmography-based respiration signal) that was not higher than $2.26 \%$ in median nor interquartile range (IQR) for all stages of fixed and spontaneous breathing, and not higher than $3.57 \%$ in median nor IQR in the case when the low computational cost algorithm was applied. These results demonstrate that respiration-related modulation of the ECG morphology are also present in the armband ECG device. Furthermore, these results suggest that respiration-related modulation can be exploited by the EDR method based on QRS slopes and R-wave angles to obtain respiratory rate, which may have a wide range of applications including monitoring patients with chronic respiratory diseases, epileptic seizures detection, stress assessment, and sleep studies, among others.
\end{abstract}

Index Terms-Wearable devices, electrocardiogram (ECG), Respiratory rate, ECG derived respiration (EDR)

\section{INTRODUCTION}

$\mathrm{R}^{\mathrm{r}}$ ESPIRATORY rate is a sensitive clinical marker in many pulmonary diseases [1], e.g., it is the first marker for acute respiratory dysfunction [2], and it is useful for detection of periodic breathing [3] which leads to a higher mortality in

This project has received funding from the European Union's Framework Programme for Research and Innovation Horizon 2020 (2014-2020) under the Marie Skłodowska-Curie Grant Agreement No. 745755. This work was also supported by Government of Aragón and European Social Fund (EU) through BSICoS group (T96), and by CIBER in Bioengineering, Biomaterials \& Nanomedicine (CIBER-BBN) through Instituto de Salud Carlos III, and by MCIU, AEI and FEDER under project RTI2018-097723-B-I00. The computation was performed by the ICTS NANBIOSIS, specifically by the High Performance Computing Unit of CIBER-BBN at University of Zaragoza. This work was also supported by NSF SBIR Phase I (\#1746589) and R43 HL135961. patients suffering from heart failure [4]. Taking into consideration the high sensitivity of respiratory rate as an indicator of serious illness, it has received little attention in the clinical field for a long time, being denominated either neglected [5] or forgotten [6] vital signs by some researchers. However, the importance of respiratory rate for the general health is recently receiving more attention [7] and its consideration in continuous monitoring is of high interest.

Multimodal analysis of respiratory rate and electrocardiogram (ECG) opens a wider range of applications including sleep studies [8], epileptic seizure detection [9], stress level assessment [10], and monitoring patients suffering from chronic respiratory diseases such as asthma [11] or chronic obstructive pulmonary disease (COPD). The chronic respiratory patients are affected by destabilizations (or "exacerbations") which makes them to seek medical help and often lead to their hospitalization. These exacerbations are one of the main causes of mortality among patients with COPD and asthma [12], [13]. Furthermore, the prognosis of the patients with COPD and asthma exacerbation depends on a timely diagnosis and treatment [12], [13]. Daily monitoring of ECG and respiratory rate could be useful for early detection of exacerbations, allowing a better prognosis of this type of patients. Thus, a wearable cardiorespiratory monitor would have a wide range of applications.

The wearable continuous ECG monitoring options in the clinical routine are Holter/event monitors and more recently developed chest-patch devices. The Holter and event monitors are cumbersome devices with obtrusive electrode leads. Furthermore, these devices use wet (hydrogel-based) electrodes, which often lead to skin irritation [14] because of the hydrogel and the adhesive needed to fix the electrodes to the skin [15]. This limits Holter monitors to be usable only for short-term monitoring (few weeks). Although the patch devices do not have the disadvantage of obstructive leads, they also use

*J. Lázaro (corresponding author), N. Reljin, and K. H. Chon are with the Biomedical Engineering Department, University of Connecticut, Storrs, CT 06269, USA (e-mail: jesus.lazaro@uconn.edu).

J. Lázaro, R. Bailón, E. Gil and P. Laguna are with the Biomedical Signal Interpretation and Computational Simulation (BSICoS) group at the Aragón Institute of Engineering Research (I3A), IIS Aragón, University of Zaragoza, Spain, and CIBER de Bioingeniería, Biomateriales y Nanomedicina (CIBER$\mathrm{BBN})$, Spain.

Y. Noh is with the College of Nursing and with the Department of Electrical and Computer Engineering of the University of Massachussetts, Amherst, MA 01003, USA. 
hydrogels and adhesives causing skin irritation when used for long periods of time. In order to overcome these disadvantages, an armband ECG monitor device is being developed in our lab at the University of Connecticut. This device consists of an armband that is worn on the left upper arm, and it records 3 ECG channels using differentially 3 pairs of dry electrodes also developed in our lab [16]. Thus, the armband setup remains a very convenient setup for long-term daily ECG monitoring since skin irritant hydrogen and adhesives are not used.

With respect to respiration monitoring approaches, they require cumbersome devices that usually interfere with natural breathing and are intractable for some applications such as sleep studies, stress tests, and monitoring normal daily living [17]. Thus, other methods for deriving respiration from other noninvasive signals have been proposed in the literature. Some of these methods use signals which can be acquired in ambulatory environments, such as ECG or pulse photoplethysmogram [18], or even smartphones [19]. Methods for obtaining respiratory rates from the ECG are also known as ECG derived respiration (EDR) methods, and they exploit different respiration-related modulations that can be observed in the ECG. On one hand, there is a well-known phenomenon referred as the respiratory sinus arrhythmia (RSA) which consists of a respirationsynchronous modulation of the heart rate [20]. However, the RSA is driven by the autonomic nervous system, thus, limits the applications based on RSA for some subjects (and thus excluding, among others, patients with atrial fibrillation and/or patients with pacemakers). On the other hand, respiration modulates the ECG morphology through two effects: first, the movement of the electrodes relative to the heart, and, second, the impedance changes in the chest due to the variation of the amount of air in the lungs during the respiratory cycle. In a previous study, a method exploiting this respiration-related modulation of the ECG morphology through the measurement of QRS slopes and R-wave angle showed good performance when evaluated with exercise stress test recordings [21], which are characterized by high non-stationarity and noise. However, the armband remains a much more challenging setup than the conventional ECG recorded by hydrogel electrodes over the chest. The armband's ECG signal-to-noise ratio is lower due to the nature of the electrodes (dry, using no hydrogel), and their close proximity to each other (all on the left upper arm). Moreover, the respiration-related movement of the left upper arm with respect to the heart is limited in comparison to the case of the chest. Therefore, the EDR methods based on ECG morphology may perform significantly worse (or even not show a significant respiratory modulation) when using the signals from the armband.

In this paper, the EDR method based on QRS slopes and Rwave angle [21] is evaluated with ECG signals recorded by the armband device. A pilot study showed promising results in 5 subjects during controlled-breathing at $0.1,0.2,0.3$, and $0.4 \mathrm{~Hz}$ [22]. The study has been further developed including 10 additional subjects (a total of 15 ) and 2 additional stages: one stage with controlled breathing at $0.5 \mathrm{~Hz}$, and another stage with spontaneous breathing. Moreover, we have modified the EDR algorithm in order to lower its computational cost so that

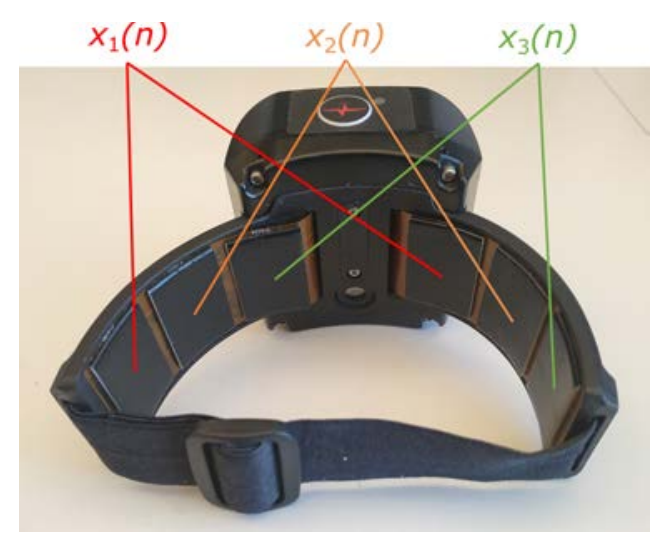

Figure 1. Photography of the wearable armband device, and the electrode configuration for the three ECG channels $x_{1}(n), x_{2}(n)$, and $x_{3}(n)$.

real-time respiratory rates can be obtained.

\section{Materials AND MethodS}

\section{A. Data acquisition and preprocessing}

Armband-ECG and respiratory signals were simultaneously recorded from 15 healthy volunteers (11 male) during a controlled breathing exercise. The experimental protocol was approved by the Institutional Review Board at the University of Connecticut (Protocol H16-107). These volunteers were sitting and asked to breathe at a constant rate guided by a video in five different stages, with each lasting for $150 \mathrm{s:} 0.1,0.2,0.3,0.4$, and $0.5 \mathrm{~Hz}$. Subsequently, the protocol included a stage with subjects breathing spontaneously in the supine position for 5 minutes. The armband used 3 pairs of carbon-black dry electrodes [16] to record 3-channel ECG with a sampling rate of $F_{\mathrm{S}}=1000 \mathrm{~Hz}$. A photo of the armband and the configuration of multi-ECG channels is shown in Fig. 1. For reference purposes, a respiratory signal was recorded by using two plethysmography-based respiration bands (Respitrace, Ambulatory Monitoring, Inc., Ardsley NY, USA), and digitalized with a 16-bit A/D converter (PowerLab/4SP, ADInstruments, Inc., Dunedin, New Zealand) at a sampling rate of $200 \mathrm{~Hz}$. The respiratory signal, which is computed by the device as the sum of the outputs of the two plethysmographic bands, was resampled to $4 \mathrm{~Hz}$.

A band-pass filter was applied to the armband signals because they contain significant EMG artifacts. The cut-off frequencies of the bandpass were set to $3 \mathrm{~Hz}$ and $25 \mathrm{~Hz}$. Note that the $3 \mathrm{~Hz}$ high-pass cut-off frequency does not distort respiratory frequencies when EDR is extracted from QRS slopes, since the QRS frequency content is well above $3 \mathrm{~Hz}$, in contrast to the baseline. The 3 band-pass filtered ECG signals are denoted as $x_{1}(n), x_{2}(n), x_{3}(n)$ in this paper. The principal component analysis (PCA) was performed on the 3-channel ECG, and the first principal component was used as the processed ECG data. This new ECG signal is denoted $x_{\mathrm{PCA}}(n)$ in this paper. An example of the 3-channel ECG signals and the $x_{\mathrm{PCA}}(n)$ can be observed in Fig. 2. 

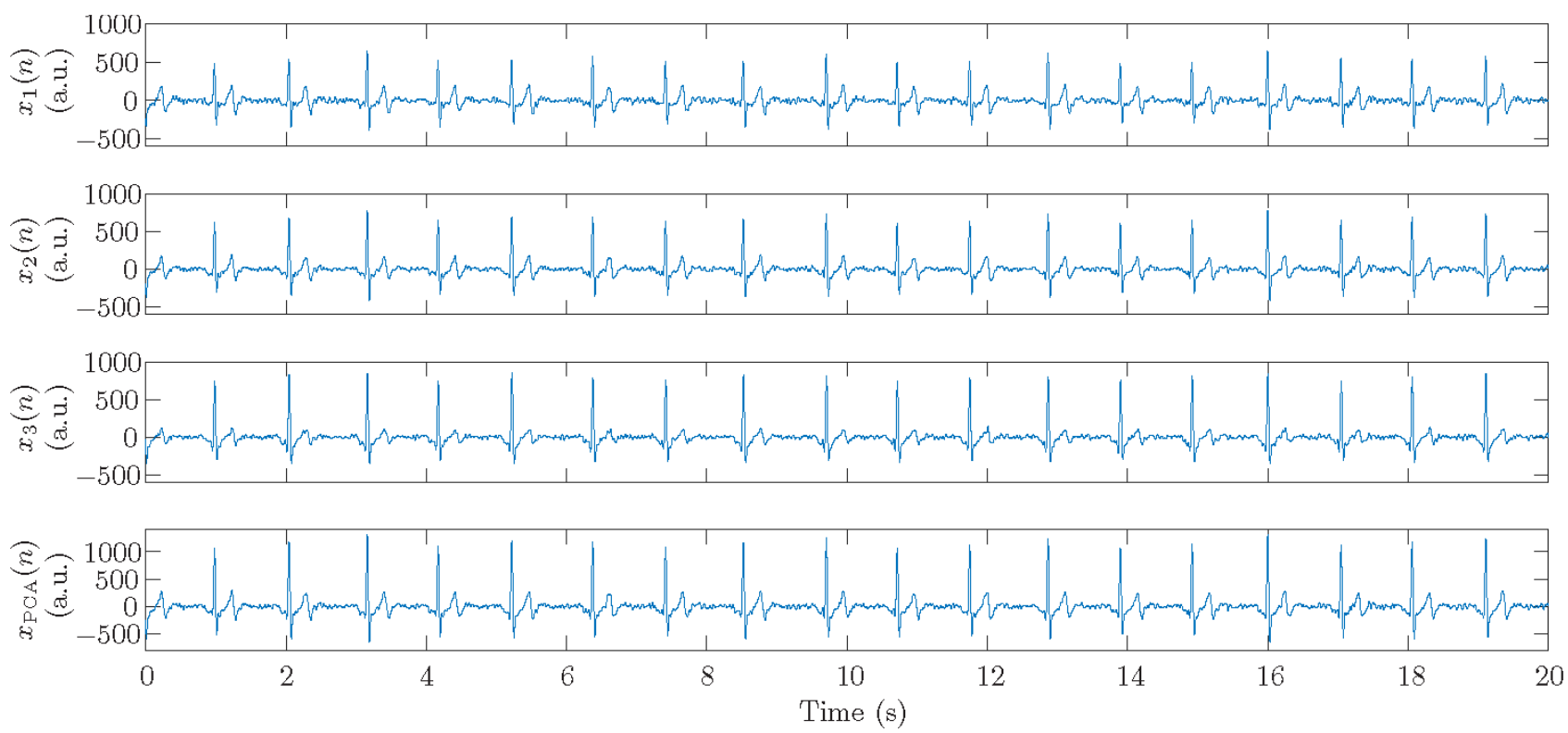

Figure 2. An example of 20 seconds of armband-ECG signals $x_{1}(n), x_{2}(n), x_{3}(n)$, and the ECG channel generated by the first component of the PCA $x_{\mathrm{PCA}}(n)$. A respiration-related modulation can be observed in the amplitude of the four shown ECG channels. In this example, subject was breathing at $0.3 \mathrm{~Hz}$.

\section{B. Electrocardiogram-derived respiration signals}

Three EDR signals were computed from each ECG channel based on beat-morphological features, specifically, the QRS slopes and the R-wave angle. This EDR technique was chosen because it was shown to be the most robust technique when tested in different scenarios [23], including highly nonstationary and noisy environments [21]. The first step of the method involves ECG delineation for obtaining Q (or QRS onset when no Q wave is present), R, and S (or QRS offset when no $\mathrm{S}$ wave is present) peaks of the $i$ th detected QRS complex $\left(n_{\mathrm{Q}_{i}}, n_{\mathrm{R}_{i}}\right.$, and $n_{\mathrm{S}_{i}}$, respectively). R peaks $\left(n_{\mathrm{R}_{i}}\right)$ were detected by the algorithm based on variable frequency complex demodulation presented in [24]. Then, for each of the ECG channels, $\mathrm{Q}$ and $\mathrm{S}$ peaks $\left(n_{\mathrm{Q}_{i}}\right.$ and $n_{\mathrm{S}_{i}}$ ) were set as the time instants where the ECG has minimum amplitude within $40 \mathrm{~ms}$ before and after $n_{\mathrm{R}_{i}}$, respectively. Subsequently, the points of maximum variation from $n_{\mathrm{Q}_{i}}$ to $n_{\mathrm{R}_{i}}$, and from $n_{\mathrm{R}_{i}}$ to $n_{\mathrm{S}_{i}}$ are computed:

$n_{\mathrm{U}_{i}}=\underset{n \in\left[n_{\mathrm{Q}_{i}}, n_{\mathrm{R}_{i}}\right]}{\operatorname{argmax}}\left\{\left|x^{\prime}(n)\right|\right\}$

$n_{\mathrm{D}_{i}}=\underset{n \in\left[n_{\mathrm{R}_{i}}, n_{\mathrm{S}_{i}}\right]}{\operatorname{argmax}}\left\{\left|x^{\prime}(n)\right|\right\}$,

where $x^{\prime}(n)$ represents the first derivative of the ECG channel. Then, a straight line is fitted to the ECG signal by the least squares in two intervals of $8 \mathrm{~ms}$ : one centered at $n_{\mathrm{U}_{i}}$, and the other centered at $n_{\mathrm{D}_{i}}$. Their slopes are denoted $I_{\mathrm{US}_{i}}$ and $I_{\mathrm{DS}}$ respectively, and they are used to measure the R-wave angle:

$\Phi_{\mathrm{R}_{i}}=\arctan \left(\frac{I_{\mathrm{US}_{i}}-I_{\mathrm{DS}_{i}}}{0.4\left(6.25+I_{\mathrm{US}_{i}} I_{\mathrm{DS}}\right)}\right)$,

which is scaled to match units in the conventional ECG tracings in clinical print-outs $(25 \mathrm{~mm} / \mathrm{s}$ and $10 \mathrm{~mm} / \mathrm{mV})$.

These features related to the QRS morphology were used to obtain an unevenly (beat by beat) sampled version of EDR signals:
$d_{\{\mathrm{US}, \mathrm{DS}, \Phi\}}^{u}(n)=\sum_{i}\left\{I_{\mathrm{US}_{i}}, I_{\mathrm{DS}_{i}}, \Phi_{\mathrm{R}_{i}}\right\} \delta\left(n-n_{\mathrm{R}_{i}}\right)$.

Later, a median-absolute-deviation-based rule was applied for outlier rejection, and a 4-Hz evenly sampled version of each series was obtained by cubic splines interpolation. Then, these evenly-sampled version of the EDR signals were band-pass filtered $[0.075,1] \mathrm{Hz}$. The resulting EDR signals are denoted without the superscript " $u$ " in this paper, i.e., $d_{\mathrm{US}}(n), d_{\mathrm{DS}}(n)$, and $d_{\Phi}(n)$. In this way, three EDR signals per ECG channel were obtained. An example of the obtained EDR signals can be observed in Fig. 3. Further details about the algorithm can be found in [21].

An additional parameter was derived from these QRS slopes by computing their differences $\left(I_{\mathrm{US}_{i}}-I_{\mathrm{DS}_{i}}\right)$. This parameter is called QRS slopes range (SR) and it was proposed in [25] for obtaining an EDR signal in the time domain that combines the information of both QRS slopes. This combination is faster than the combination proposed in [21] (see Section II-C), hence, SR may be more appropriate for wearables such as the armband from the point of view of computational time. A new EDR signal was obtained based on $\mathrm{SR}$, and it is denoted $d_{\mathrm{SR}}(n)$ in this paper.

In this work, a low-computational-cost (LC) version of the algorithm was developed. First, $x_{1}(n), x_{2}(n), x_{3}(n)$ were downsampled to $250 \mathrm{~Hz}$, and the $x_{\mathrm{PCA}}(n)$ was also sampled at $250 \mathrm{~Hz}$. Subsequently, QRS complexes $\left(n_{\mathrm{QRS}}\right)$ were detected in $x_{\mathrm{PCA}}(n)$ by the algorithm based on the variable frequency complex demodulation described in [24]. Then, for each of the ECG channels, $n_{\mathrm{R}_{i}}^{\mathrm{LC}}$ was set as the time instant when the ECG has maximum amplitude within $80 \mathrm{~ms}$ centered in $n_{\mathrm{QRS}_{i}}$ (note that, $n_{\mathrm{QRS}_{i}}$ and $n_{\mathrm{R}_{i}}^{\mathrm{LC}}$ are the same for $x_{\mathrm{PCA}}(n)$ ), and Q and S peaks ( $n_{\mathrm{Q}_{i}}^{\mathrm{LC}}$ and $n_{\mathrm{S}_{i}}^{\mathrm{LC}}$ ) were set as the time instants where the ECG has minimum amplitude within 40 ms before and after $n_{\mathrm{R}_{i}}^{\mathrm{LC}}$, respectively. Subsequently, the $\mathrm{LC}$ version of QRS slopes $\left(\mathrm{IUS}_{i}^{\mathrm{LC}}\right.$ 

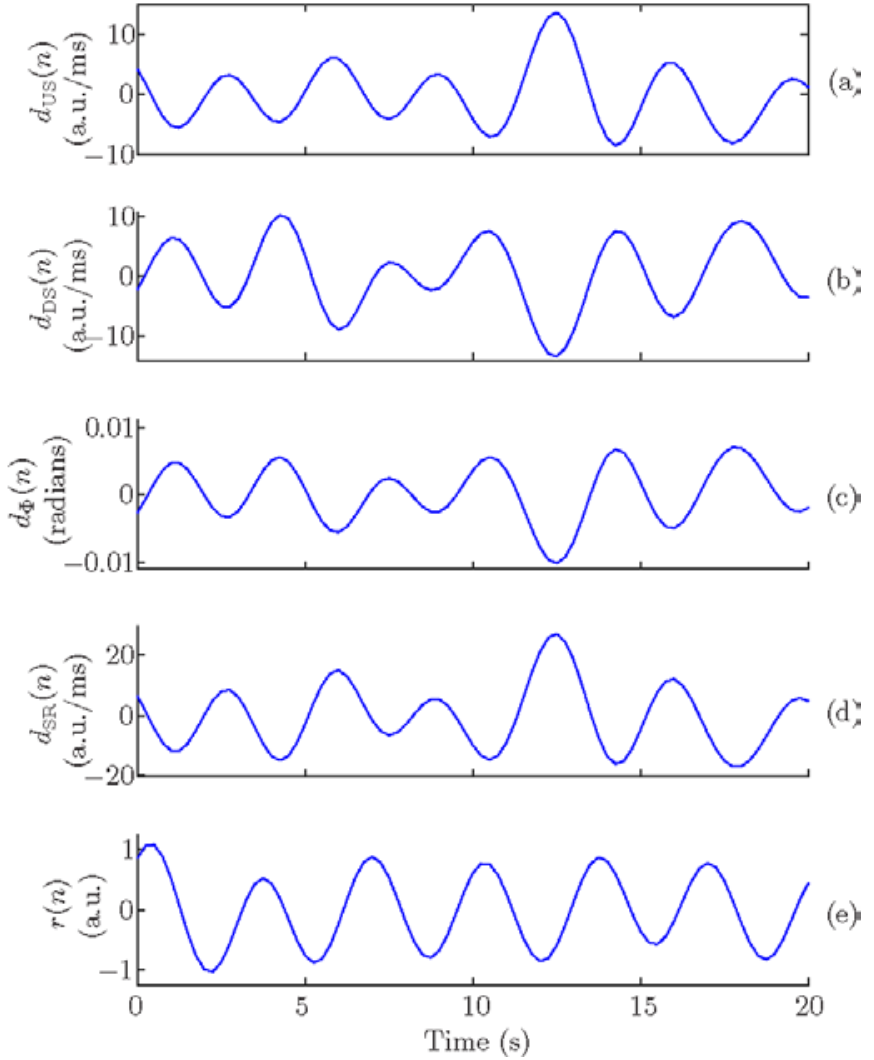

Figure 3. Example of 20 seconds of EDR signals from $x_{\mathrm{PCA}}(n)$ : (a) $d_{\mathrm{US}}(n)$, (b) $d_{\mathrm{DS}}(n)$, (c) $d_{\Phi}(n)$, and (d) $d_{\mathrm{SR}}(n)$. Reference respiratory signal $r(n)$ is also shown for visual comparison (e). In this example, subject was breathing at 0.3 $\mathrm{Hz}$.

and $I_{\mathrm{DS}}^{\mathrm{LC}}$ ) were computed as the maximum of the first derivative of studied ECG signal with no posterior line fitting [25]:

$I_{\mathrm{US}_{i}}^{\mathrm{LC}}=\max _{n \in\left[n_{\mathrm{Q}_{i}}^{\mathrm{LC}}, n_{\mathrm{R}_{i}}^{\mathrm{LC}}\right]}\left\{\left|x^{\prime}(n)\right|\right\}$,

$I_{\mathrm{DS}_{i}}^{\mathrm{LC}}=\max _{n \in\left[n_{\mathrm{R}_{i}}^{\mathrm{LC}}, n_{\mathrm{S}_{i}}^{\mathrm{LC}}\right]}\left\{\left|x^{\prime}(n)\right|\right\}$.

The LC version of QRS slopes range and the R-wave angle $\Phi_{\mathrm{R}_{i}}^{\mathrm{LC}}$ was computed, this time using $I_{\mathrm{US}}^{\mathrm{LC}}$ and $I_{\mathrm{DS}}^{\mathrm{LC}}$. Then, LCEDR signals were computed from these LC features (QRS slopes range and R-wave angle) by a similar procedure to the non-LC case, i.e., consisting of the median-absolute-deviation outlier rejection rule, cubic splines interpolation, and band-pass filtering. Therefore, two LC-EDR signals were obtained per ECG channel: one based on QRS slopes range $\left(d_{\mathrm{SR}}^{\mathrm{LC}}(n)\right)$, and the other based on R-wave angle $\left(d_{\Phi}^{\mathrm{LC}}(n)\right)$. Figure 4 shows an example of a QRS complex with its corresponding fitted straight lines from where $I_{\mathrm{US}_{i}}$ and $I_{\mathrm{DS}_{i}}$ are computed, and, in addition, it shows the first derivative of the 250-Hz-sampled version of the signal, from which $I_{\mathrm{US}}^{\mathrm{LC}}$ and $I_{\mathrm{DS}}^{\mathrm{LC}}$ are computed.

\section{Respiratory rate estimation}

The respiratory rate was estimated by using a time-frequency algorithm which can offer an estimate using information from several EDR signals [21]. The first step of the algorithm is the computation of power spectrum for the $j$ th EDR signal and the $k$ th running interval of $42 \mathrm{~s}\left(S_{j}(k, f)\right)$. The $S_{j}(k, f)$ is obtained by the Welch periodogram, using windows of $12 \mathrm{~s}$ with $50 \%$ overlap. A $S_{j}(k, f)$ was generated at each $5 \mathrm{~s}$, and the
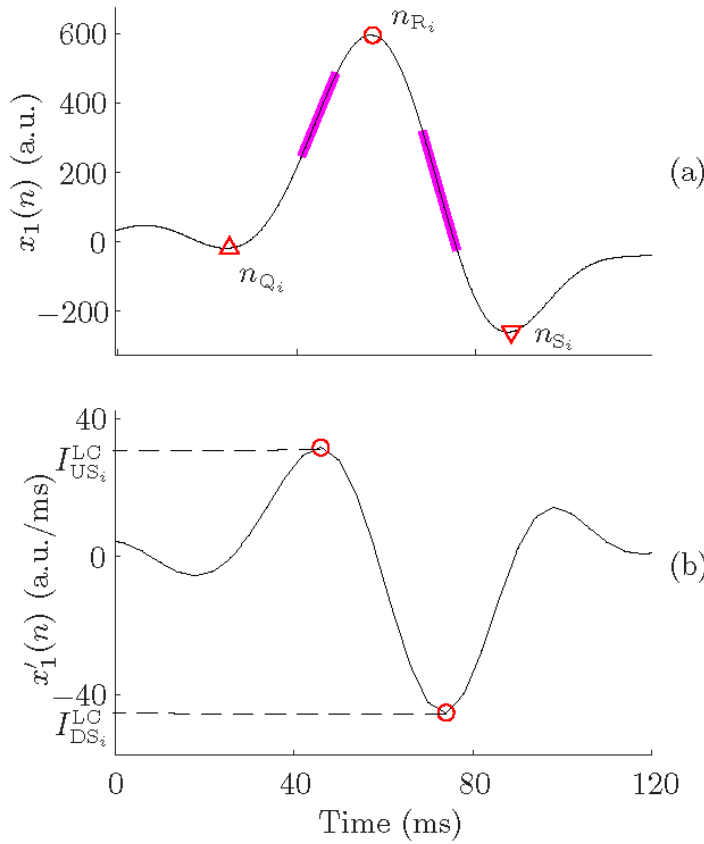

Figure 4. (a) Example of QRS complex in $x_{1}(n)$ and its corresponding $n_{\mathrm{Q}_{i}}, n_{\mathrm{R}_{i}}$, and $n_{\mathrm{S}_{i}}$. The fitted straight lines from where the QRS slopes $\left(I_{\mathrm{US}_{i}}\right.$ and $\left.I_{\mathrm{DS}}\right)$ are estimated, are shown in magenta. (b) In addition, the first derivative of the 250Hz-sampled version of $x_{1}(n)$, where the LC estimations of QRS slopes ( $I_{\mathrm{US}_{i}}^{\mathrm{LC}}$ and $I_{\mathrm{DS}_{i}}^{\mathrm{LC}}$ ) are computed.

concatenation of all segments resulted in a time-frequency power spectrum.

The second step of the algorithm is a peak-conditioned average of normalized-in-power $S_{j}(k, f)$, denoted $\bar{S}(k, f)$ in this paper. A $\bar{S}(k, f)$ is generated each $5 \mathrm{~s}$ from the $S_{j}(k, f)$ of the surrounding $25 \mathrm{~s}$. Thus, up to five $S_{j}(k, f)$ per each used EDR signal can take part in $\bar{S}(k, f)$. However, only those power spectra having a "peaked" shape are chosen. The decision of whether a $S_{j}(k, f)$ has a peaked shape or not is based on the power around a peak with respect to the total power within the band where respiration is expected (a time-varying sub-band within $[0.075,1] \mathrm{Hz}$ which depends on the previous estimates). Note that in time instants where none of the qualifying $S_{j}(k, f)$ does has a peaked shape, the respiratory rate would not be estimated at that specific time. This algorithm is described in more detail in [21].

Respiratory rate, $\hat{f}_{\mathrm{RES}}(k)$, was estimated every $5 \mathrm{~s}$ during each one of the stages of the protocol $(0.1 \mathrm{~Hz}, 0.2 \mathrm{~Hz}, 0.3 \mathrm{~Hz}$, $0.4 \mathrm{~Hz}, 0.5 \mathrm{~Hz}$, and spontaneous breathing) from the three combinations consisting of:

- $\quad C_{3 \mathrm{ECG}}$ : The three EDR signals $\left(d_{\mathrm{US}}(n), d_{\mathrm{DS}}(n)\right.$, and $\left.d_{\Phi}(n)\right)$ from $x_{1}(n), x_{2}(n)$, and $x_{3}(n)$ (9 EDR signals).

- $\quad C_{\mathrm{PCA}}:$ The three EDR signals $\left(d_{\mathrm{US}}(n), d_{\mathrm{DS}}(n)\right.$, and $\left.d_{\Phi}(n)\right)$ from $x_{\mathrm{PCA}}(n)$ (3 EDR signals).

- $\quad C_{\mathrm{ALL}}$ : All the EDR signals in $C_{\text {3ECG }}$ or in $C_{\mathrm{PCA}}$ (12 EDR signals).

An example of the obtained $\bar{S}(k, f)$ during spontaneous breathing can be observed in Fig. 5. Similar combinations based on the slope range approach were computed in order to assess the difference in performance of such approach and the original methods in [21]. 

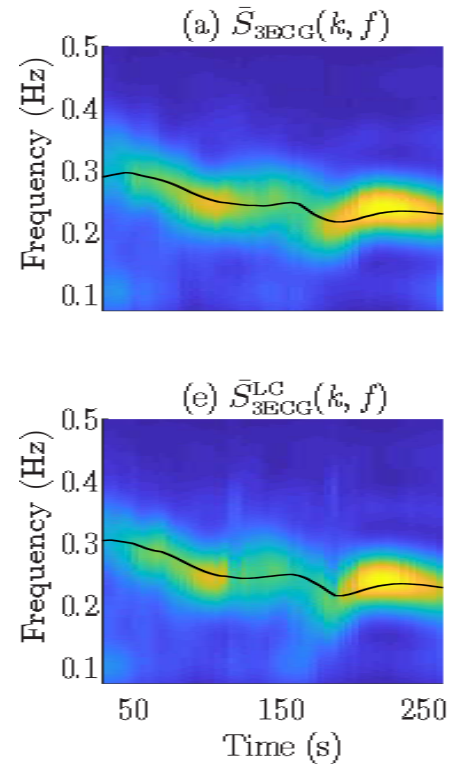

(b) $\bar{S}_{\mathrm{PCA}}(k, f)$

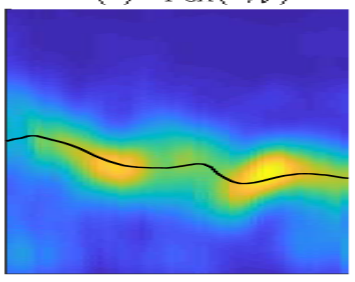

(f) $\bar{S}_{\mathrm{PCA}}^{\mathrm{LG}}(k, f)$

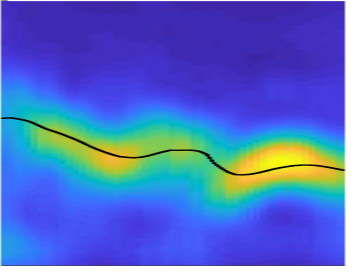

50
150 Time (s)

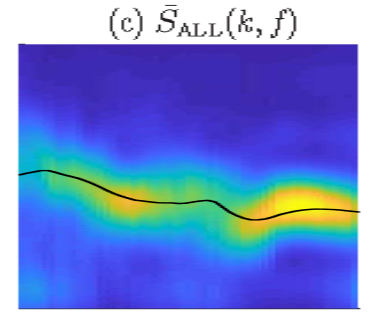

(g) $\bar{S}_{A \mathrm{LL}}^{\mathrm{L} C}(k, f)$

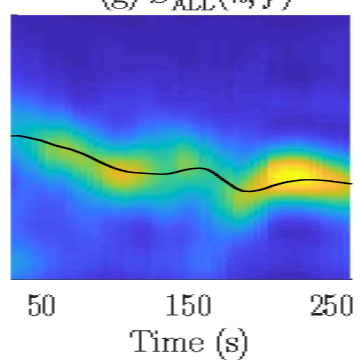

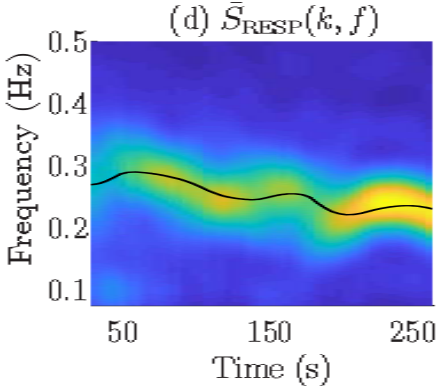

Time (s)

Figure 5. Example of peak-conditioned average $\bar{S}(k, f)$ obtained from the combinations during spontaneous breathing: (a) $C_{3 \mathrm{ECG}}$, (b) $C_{\mathrm{PCA}}$, and (c) $C_{\mathrm{ALL}} . \bar{S}(k, f)$ obtained from the reference respiratory rate is also shown for visual comparison (d). In addition, the $\bar{S}(k, f)$ obtained from the LC version of the methods are also shown: (e) $C_{3 \mathrm{ECG}}^{\mathrm{LC}}$, (f) $C_{\mathrm{PCA}}^{\mathrm{LC}}$, and (g) $C_{\mathrm{ALL}}^{\mathrm{LC}}$.

- $\quad C_{3 \mathrm{ECG}}^{\mathrm{SR}}$ : The two LC-EDR signals $\left(d_{\mathrm{SR}}(n)\right.$ and $\left.d_{\Phi}(n)\right)$ from $x_{1}(n), x_{2}(n)$, and $x_{3}(n)$ (6 EDR signals).

- $\quad C_{\mathrm{PCA}}^{\mathrm{SR}}$ : The two EDR signals $\left(d_{\mathrm{SR}}(n)\right.$ and $\left.d_{\Phi}(n)\right)$ from $X_{\mathrm{PCA}}(n)$ (2 EDR signals).

- $C_{\mathrm{ALL}}^{\mathrm{SR}}$ : All the EDR signals in $C_{3 \mathrm{ECG}}$ or in $C_{\mathrm{PCA}}$ (8 EDR signals).

In addition, similar combinations were used for combining the LC-EDR signals:

- $\quad C_{3 \mathrm{ECG}}^{\mathrm{LC}}$ : The two LC-EDR signals $\left(d_{\mathrm{SR}}^{\mathrm{LC}}(n)\right.$ and $\left.d_{\Phi}^{\mathrm{LC}}(n)\right)$ from $x_{1}(n), x_{2}(n)$, and $x_{3}(n)$ (6 EDR signals).

- $\quad C_{\mathrm{PCA}}^{\mathrm{LC}}$ : The two EDR signals $\left(d_{\mathrm{SR}}^{\mathrm{LC}}(n)\right.$ and $\left.d_{\Phi}^{\mathrm{LC}}(n)\right)$ from $x_{\mathrm{PCA}}(n)$ (2 EDR signals).

- $\quad C_{\mathrm{ALL}}^{\mathrm{LC}}$ : All the EDR signals in $C_{3 \mathrm{ECG}}^{\mathrm{LC}}$ or in $C_{\mathrm{PCA}}^{\mathrm{LC}}(8 \mathrm{EDR}$ signals).

The reference respiratory rates, $f_{\mathrm{RES}}(k)$, were derived from the plethysmography-based respiratory signals using the same algorithm. Relative error of the respiratory rate estimations with respect to the reference respiratory rate was computed for each subject and stage of the protocol as:

$e(k)=\frac{\hat{f}_{\mathrm{RES}}(k)-f_{\mathrm{RES}}(k)}{f_{\mathrm{RES}}(k)} \times 100$.

The Friedman statistical test and the Bonferroni correction were used for analysis of differences of $e(k)$ for the different methods. The paired non-parametric Friedman statistical test was chosen because it was observed that $e(k)$ were not normal distributed, and the Bonferroni correction was applied in order to control the familywise error rate because multiple comparisons were performed.

\section{RESULTS}

There were no periods when none of the qualifying $S_{j}(k, f)$ has a peaked shape, so respiratory rate could be estimated in all the cases for all subjects.

Two out of the 15 subjects showed a mean heart rate lower than twice the respiratory rate when they breathe at $0.5 \mathrm{~Hz}$. These two subjects' results were not taken into account for the respiration rate at $0.5 \mathrm{~Hz}$. The reason for this exclusion is that respiratory information is obtained only at the beat occurrence, i.e., its intrinsic sampling rate equals to the heart rate. Therefore, the highest trackable frequency is half of the mean heart rate due to Nyquist theorem.

Table I shows the inter-subject median of the intra-subject median and interquartile range (IQR) of $e(k)$ for each studied combination of EDR signals, and for each stage of the protocol. In addition, Table II shows the percentage of respiratory rate estimates for which $e(k)$ was less than $5 \%\left(\mathrm{R}_{<5 \%}\right)$, and the percentage of respiratory rate estimates for which $e(k)$ was less than $3 \%\left(\mathrm{R}_{<3 \%}\right)$ are also shown. Figure 6 shows regression plots and Bland-Altman plots for the best methods in terms of accuracy and computation time. The significant differences $(p<0.05)$ found by the Friedman statistical test are shown in Table III.

Moreover, the mean computational time per minute of signal, using Matlab R2017b on an Intel Core I7 4770, is shown in Table IV for each one of the studied methods.

\section{DISCUSSION}

An EDR algorithm has been evaluated for respiratory rate estimation derived from a wearable armband ECG monitor. The armband device was developed in our lab at the University of Connecticut, and it records three-channel ECG signals using three pairs of dry electrodes. This ECG device is much more convenient for a daily monitoring because it does not use obstructive leads nor wet (hydrogel) electrodes, in contrast to the conventional Holter monitor. A pilot study was presented in [22]. The study was further developed by including 10 additional subjects (a total of 15), monitoring breathing using both controlled breathing and spontaneous breathing. We have also modified the EDR algorithm in order to lower its computational cost.

The original algorithm in [21] obtained very promising results with medians and IQRs of relative error with respect to the reference signal not greater than $2.26 \%$ for all stages when 


\begin{tabular}{|c|c|c|c|c|c|c|c|}
\hline & & \multicolumn{2}{|c|}{ Original methods in [20] } & \multicolumn{2}{|c|}{$\begin{array}{l}\text { Slopes range and R-wave } \\
\text { angle methods }\end{array}$} & \multicolumn{2}{|c|}{ LC methods } \\
\hline & & $\begin{array}{c}\text { Median of } \\
\text { medians }\end{array}$ & $\begin{array}{l}\text { Median of } \\
\text { IQRs }\end{array}$ & $\begin{array}{c}\text { Median of } \\
\text { medians }\end{array}$ & $\begin{array}{l}\text { Median of } \\
\text { IQRs }\end{array}$ & $\begin{array}{c}\text { Median of } \\
\text { medians }\end{array}$ & $\begin{array}{l}\text { Median of } \\
\text { IQRs }\end{array}$ \\
\hline \multirow{3}{*}{$0.1 \mathrm{~Hz}$} & $C_{3 \text { ECG }}$ & $1.40 \%$ & $1.11 \%$ & $1.43 \%$ & $0.72 \%$ & $1.12 \%$ & $1.90 \%$ \\
\hline & $C_{\text {PCA }}$ & $0.65 \%$ & $1.33 \%$ & $1.21 \%$ & $0.77 \%$ & $0.27 \%$ & $1.68 \%$ \\
\hline & $C_{\mathrm{ALL}}$ & $1.39 \%$ & $1.15 \%$ & $1.00 \%$ & $0.72 \%$ & $0.61 \%$ & $1.72 \%$ \\
\hline \multirow{3}{*}{$0.2 \mathrm{~Hz}$} & $C_{3 \mathrm{ECG}}$ & $-0.08 \%$ & $0.42 \%$ & $0.20 \%$ & $0.33 \%$ & $-0.17 \%$ & $0.57 \%$ \\
\hline & $C_{\mathrm{PCA}}$ & $0.13 \%$ & $0.47 \%$ & $0.14 \%$ & $0.33 \%$ & $0.07 \%$ & $0.38 \%$ \\
\hline & $C_{\mathrm{ALL}}$ & $-0.15 \%$ & $0.47 \%$ & $0.13 \%$ & $0.31 \%$ & $-0.45 \%$ & $0.49 \%$ \\
\hline \multirow{3}{*}{$0.3 \mathrm{~Hz}$} & $C_{3 E C G}$ & $0.07 \%$ & $0.43 \%$ & $0.08 \%$ & $0.59 \%$ & $0.04 \%$ & $0.90 \%$ \\
\hline & $C_{\text {PCA }}$ & $-0.05 \%$ & $0.64 \%$ & $0.04 \%$ & $0.55 \%$ & $-0.01 \%$ & $0.66 \%$ \\
\hline & $C_{\mathrm{ALL}}$ & $0.07 \%$ & $0.43 \%$ & $0.15 \%$ & $0.59 \%$ & $0.02 \%$ & $0.74 \%$ \\
\hline \multirow{3}{*}{$0.4 \mathrm{~Hz}$} & $C_{3 E C G}$ & $0.00 \%$ & $0.35 \%$ & $0.00 \%$ & $0.39 \%$ & $-0.21 \%$ & $0.77 \%$ \\
\hline & $C_{\mathrm{PCA}}$ & $0.03 \%$ & $0.42 \%$ & $0.00 \%$ & $0.43 \%$ & $0.04 \%$ & $0.44 \%$ \\
\hline & $C_{\mathrm{ALL}}$ & $0.00 \%$ & $0.41 \%$ & $-0.01 \%$ & $0.41 \%$ & $-0.05 \%$ & $0.36 \%$ \\
\hline \multirow{3}{*}{$0.5 \mathrm{~Hz}$} & $C_{3 E C G}$ & $-0.24 \%$ & $0.39 \%$ & $0.04 \%$ & $0.29 \%$ & $-0.36 \%$ & $0.46 \%$ \\
\hline & $C_{\mathrm{PCA}}$ & $-0.12 \%$ & $0.34 \%$ & $-0.05 \%$ & $0.38 \%$ & $-0.21 \%$ & $0.37 \%$ \\
\hline & $C_{\mathrm{ALL}}$ & $-0.20 \%$ & $0.37 \%$ & $0.00 \%$ & $0.44 \%$ & $-0.23 \%$ & $0.32 \%$ \\
\hline \multirow{3}{*}{ Spontaneous } & $C_{3 \mathrm{ECG}}$ & $-0.26 \%$ & $3.06 \%$ & $-0.15 \%$ & $3.20 \%$ & $-0.09 \%$ & $6.46 \%$ \\
\hline & $C_{\mathrm{PCA}}$ & $-0.25 \%$ & $6.66 \%$ & $-0.32 \%$ & $3.63 \%$ & $0.04 \%$ & $3.57 \%$ \\
\hline & $C_{\mathrm{ALL}}$ & $-0.03 \%$ & $2.26 \%$ & $-0.19 \%$ & $2.51 \%$ & $-0.05 \%$ & $4.59 \%$ \\
\hline
\end{tabular}

Table I. Inter-subject medians of intra-subject median and interquartile range (IQR) of obtained $e(k)$ for each studied combination of EDR signals and each stage of the protocol.

\begin{tabular}{|c|c|c|c|c|c|c|c|}
\hline & & \multicolumn{2}{|c|}{ Original methods in [20] } & \multicolumn{2}{|c|}{$\begin{array}{l}\text { Slopes range and R-wave } \\
\text { angle methods }\end{array}$} & \multicolumn{2}{|c|}{ LC methods } \\
\hline & & $\mathrm{R}_{<5 \%}$ & $\mathrm{R}_{<3 \%}$ & $\mathrm{R}_{<5 \%}$ & $\mathrm{R}_{<3 \%}$ & $\mathrm{R}_{<5 \%}$ & $\mathrm{R}_{<3 \%}$ \\
\hline \multirow{3}{*}{$0.1 \mathrm{~Hz}$} & C3ECG & $93.33 \%$ & $75.79 \%$ & $90.27 \%$ & $80.53 \%$ & $85.96 \%$ & $64.56 \%$ \\
\hline & $C_{\mathrm{PCA}}$ & $92.98 \%$ & $77.54 \%$ & $90.27 \%$ & $74.78 \%$ & $92.63 \%$ & $70.88 \%$ \\
\hline & $C_{\mathrm{ALL}}$ & $90.53 \%$ & $72.28 \%$ & $91.15 \%$ & $81.42 \%$ & $86.67 \%$ & $65.26 \%$ \\
\hline \multirow{3}{*}{$0.2 \mathrm{~Hz}$} & $C_{3 E C G}$ & $100.00 \%$ & $100.00 \%$ & $100.00 \%$ & $100.00 \%$ & $94.04 \%$ & $92.98 \%$ \\
\hline & СРСA & $98.25 \%$ & $97.54 \%$ & $97.36 \%$ & $96.48 \%$ & $97.89 \%$ & $97.54 \%$ \\
\hline & $C_{\mathrm{ALL}}$ & $100.00 \%$ & $100.00 \%$ & $100.00 \%$ & $100.00 \%$ & $97.54 \%$ & $96.84 \%$ \\
\hline \multirow{3}{*}{$0.3 \mathrm{~Hz}$} & $C_{3 E C G}$ & $100.00 \%$ & $100.00 \%$ & $100.00 \%$ & $100.00 \%$ & $89.12 \%$ & $86.32 \%$ \\
\hline & $C_{\mathrm{PCA}}$ & $93.33 \%$ & $92.28 \%$ & $93.42 \%$ & $92.54 \%$ & $92.98 \%$ & $91.93 \%$ \\
\hline & $C_{\text {ALL }}$ & $100.00 \%$ & $100.00 \%$ & $100.00 \%$ & $100.00 \%$ & $92.98 \%$ & $90.53 \%$ \\
\hline \multirow{3}{*}{$0.4 \mathrm{~Hz}$} & $C_{3 \mathrm{ECG}}$ & $93.33 \%$ & $90.53 \%$ & $92.48 \%$ & $91.59 \%$ & $64.21 \%$ & $62.46 \%$ \\
\hline & $C_{\mathrm{PCA}}$ & $90.53 \%$ & $89.82 \%$ & $91.59 \%$ & $91.15 \%$ & $83.86 \%$ & $83.86 \%$ \\
\hline & $C_{\text {ALL }}$ & $93.33 \%$ & $91.23 \%$ & $92.04 \%$ & $91.15 \%$ & $75.79 \%$ & $73.68 \%$ \\
\hline \multirow{3}{*}{$0.5 \mathrm{~Hz}$} & $C_{3 \mathrm{ECG}}$ & $94.74 \%$ & $94.33 \%$ & $97.45 \%$ & $96.94 \%$ & $75.30 \%$ & $74.90 \%$ \\
\hline & $C_{\mathrm{PCA}}$ & $88.26 \%$ & $87.45 \%$ & $88.27 \%$ & $88.27 \%$ & $76.92 \%$ & $76.11 \%$ \\
\hline & $C_{\mathrm{ALL}}$ & $94.74 \%$ & $94.33 \%$ & $89.80 \%$ & $88.78 \%$ & $75.30 \%$ & $74.90 \%$ \\
\hline \multirow{3}{*}{ Spontaneous } & $C_{3 E C G}$ & $74.83 \%$ & $67.48 \%$ & $76.15 \%$ & $70.34 \%$ & $65.44 \%$ & $55.78 \%$ \\
\hline & $C_{\mathrm{PCA}}$ & $67.89 \%$ & $61.22 \%$ & $70.34 \%$ & $62.30 \%$ & $71.70 \%$ & $62.31 \%$ \\
\hline & $C_{\mathrm{ALL}}$ & $74.83 \%$ & $67.62 \%$ & $76.75 \%$ & $71.09 \%$ & $69.39 \%$ & $58.50 \%$ \\
\hline
\end{tabular}

Table II. Percentage of estimates for which $e(k)$ was less than $5 \%\left(\mathrm{R}_{<5 \%}\right)$ and the percentage of respiratory rate estimates for which $e(k)$ was less than $3 \%\left(\mathrm{R}_{<3 \%}\right)$ are also shown.

using $C_{\mathrm{ALL}}$. Furthermore, $\mathrm{R}_{<5 \%}$ and $\mathrm{R}_{<3 \%}$ obtained when using $C_{\text {ALL }}$ were no lower than $74.83 \%$ and $67.62 \%$, respectively, in all cases. These results demonstrate that the respiration-related modulation in the ECG can be accurately derived with the armband even if it does not use electrodes over the chest. Thus, our results suggest that the respiratory-related modulation of the ECG morphology can be exploited by the method based on QRS slopes and R-wave angles to derive respiratory rate from the armband-ECG signals. These results are even better than those obtained in [21], where a relative error of $0.50 \% \pm 4.11 \%$ (mean \pm standard deviation) was obtained using conventional ECG signals during restricted-movement conditions. The reason of this observation may be that in [21], the protocol includes the abrupt cardiovascular changes that a tilt table test creates. Futures studies may include evaluation of the armband in this kind of scenario.

The $C_{3 E C G}$ obtained the highest $\mathrm{R}_{<5 \%}$ in all six stages, while $C_{\mathrm{ALL}}$ obtained it in five stages, and $C_{\mathrm{PCA}}$ in none of the stages. Regarding $\mathrm{R}_{<3 \%}, C_{3 \mathrm{ECG}}$ obtained the highest value in three stages, while $C_{\mathrm{ALL}}$ obtained it in five stages, and $C_{\mathrm{PCA}}$ in only one stage. However, $C_{\mathrm{ALL}}$ obtained similar results than $C_{3 \mathrm{ECG}}$ in every stage. These results suggest that the information of EDR signals from $X_{\mathrm{PCA}}(n)$ is redundant with the information of EDR signals from the original ECG channels, at least in a big part, for the original method in [21]. Thus, taking into consideration that the mean computational time per minute of $C_{3 \text { ECG }}$ was 

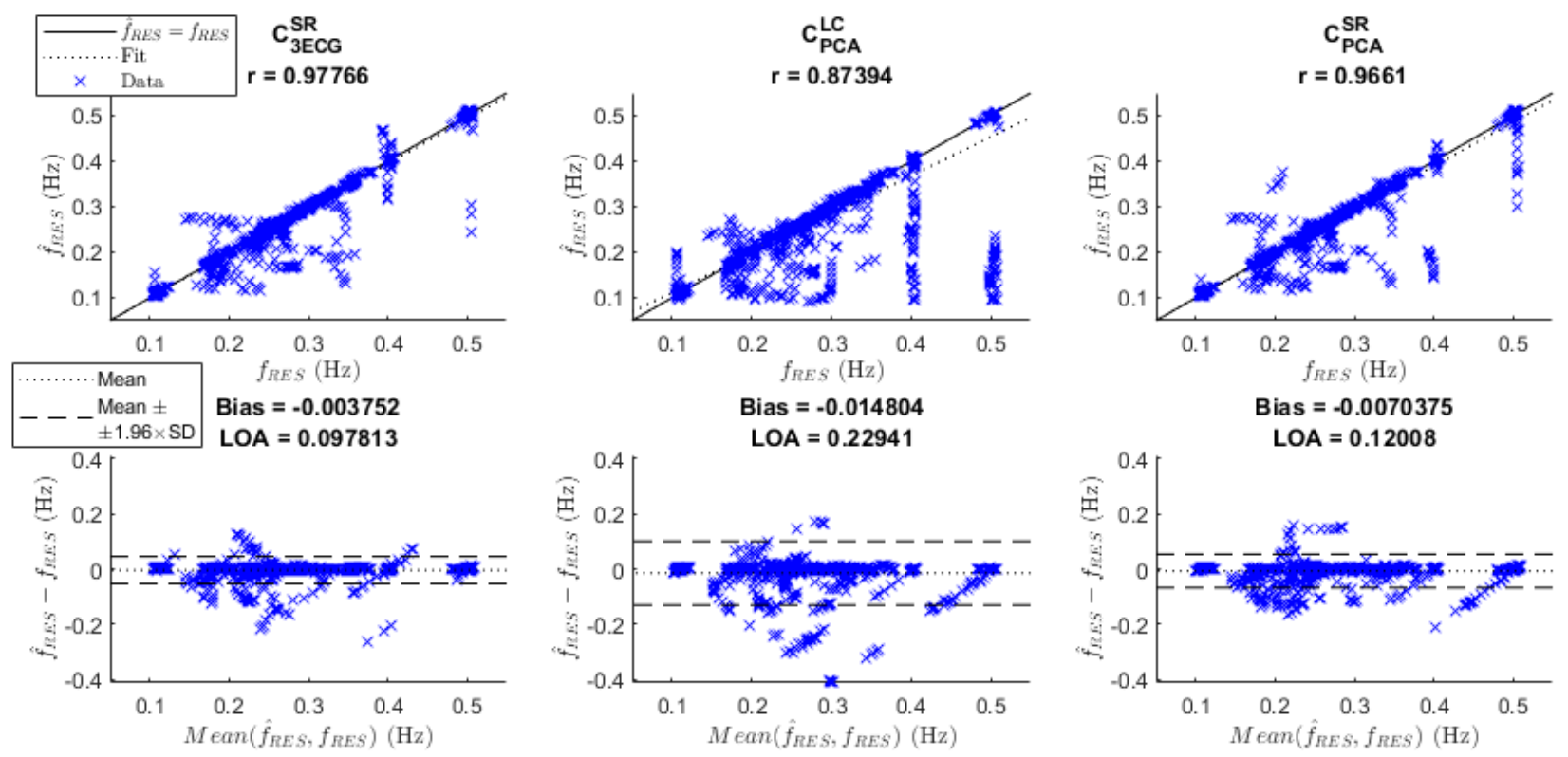

Figure 6. Regression plots (top row) and Bland-Altman plots (bottom row) for $C_{3 \mathrm{ECG}}^{\mathrm{SR}}, C_{\mathrm{PCA}}^{\mathrm{LC}}$, and $C_{\mathrm{PCA}}^{\mathrm{SR}}$. Correlations (r) are given at the top of regression plots, and bias and limits of agreement (LOA, computed as twice $2 \times 1.96 \times \mathrm{SD}$ ) are shown at top of Bland-Altman plots.

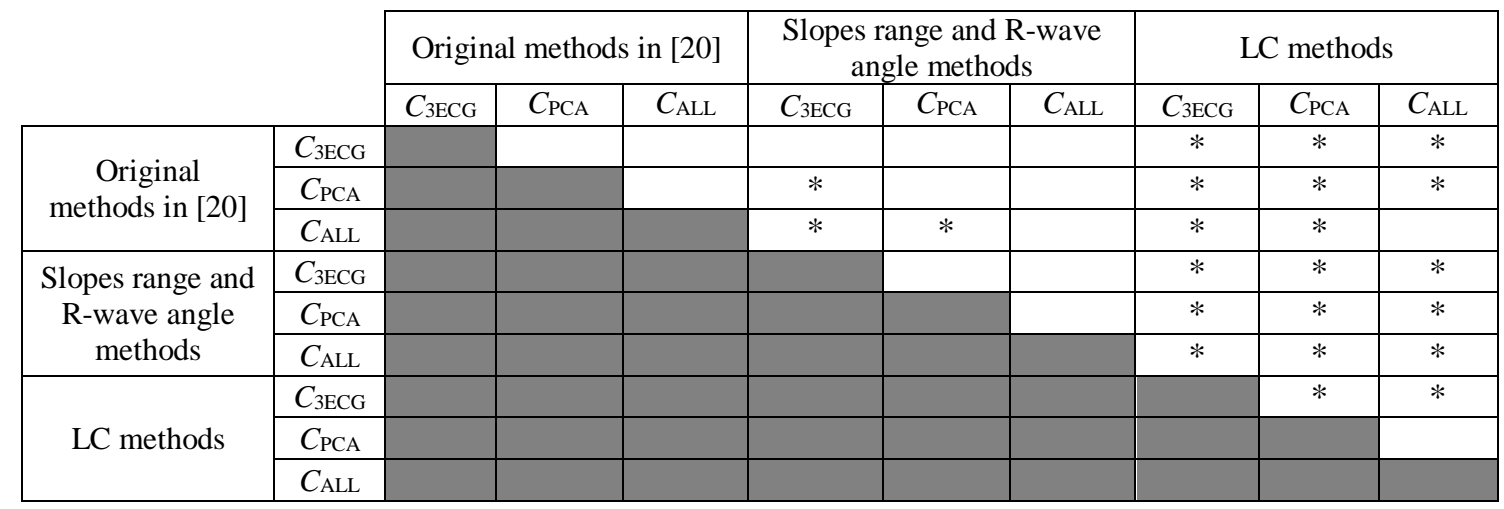

Table III. Significant differences found by the Freidman statistical test.

\begin{tabular}{|c|c|c|c|}
\hline & $\begin{array}{c}\text { Original } \\
\text { methods in } \\
{[20]}\end{array}$ & $\begin{array}{c}\text { Slopes range } \\
\text { and R-wave } \\
\text { angle methods }\end{array}$ & LC methods \\
\hline$C_{3 \mathrm{ECG}}$ & $4519.52 \mathrm{~ms}$ & $4299.71 \mathrm{~ms}$ & $1076.89 \mathrm{~ms}$ \\
\hline$C_{\text {PCA }}$ & $1166.18 \mathrm{~ms}$ & $1111.58 \mathrm{~ms}$ & $988.53 \mathrm{~ms}$ \\
\hline$C_{\text {ALL }}$ & $4645.42 \mathrm{~ms}$ & $4426.73 \mathrm{~ms}$ & $1088.29 \mathrm{~ms}$ \\
\hline
\end{tabular}

Table IV. Mean computation time per minute of signal using Matlab R2017b on an Intel Core I7 4470.

slightly lower than that of $C_{\mathrm{ALL}}$ (a $2.77 \%$ lower, $4378.23 \mathrm{~ms}$ vs. $4502.92 \mathrm{~ms}$ ), our recommendation is to use the $C_{3 \mathrm{ECG}}$ set.

Alternatively, the approach based on QRS slopes range proposed in [25] for obtaining a combined EDR signal in the time domain was also used. It consist of the unconditional subtraction of $d_{\mathrm{DS}}(n)$ to $d_{\mathrm{US}}(n)$ EDR signals. This approach is interesting in this context because it avoids the computation of one $S_{j}(k, f)$ and its peakness assessment. As $d_{\mathrm{US}}(n)$ and $d_{\mathrm{DS}}(n)$ are counterphase, this approach is very similar to combine them with the peak-conditioned average as proposed in [21]. Note that both approaches $\left(d_{\mathrm{US}}(n), d_{\mathrm{DS}}(n)\right.$, and $d_{\Phi}(n)$ vs. $d_{\mathrm{SR}}(n)$ and $d_{\Phi}(n)$, combined by peakness-conditioned average in both cases) would lead to identical results if the oscillations of
$d_{\mathrm{US}}(n)$ and $d_{\mathrm{DS}}(n)$ had similar power and they were peaked enough in the same time intervals. Results were similar to those obtained for the original methods in [21], in terms of both intersubject medians of intra-subject median and IQR of $e(k)$ (see Table I), and of $\mathrm{R}_{<5 \%}$ and $\mathrm{R}_{<3 \%}$ (see Table II). These results suggest that two approaches to combine QRS slopes information (the peak-conditioned average proposed in [21] and the unconditional subtraction proposed in [25]) lead to a similar respiratory rate estimation. In fact, the Friedman test did not find significant differences between $e(k)$ obtained from the original methods in [21] and their analogous SR methods (see Table III). Furthermore, the SR approach obtained slightly better results during spontaneous respiration. Thus, the use of QRS slopes range is preferred, as it offers similar accuracy with a slightly lower computational time $\left(4.87 \%\right.$ for $C_{3 \mathrm{ECG}}$, from $4519.92 \mathrm{~ms}$ to $4299.71 \mathrm{~ms}$ per minute of signal). In this way, our recommendation for accurate respiratory rate estimation is to use $C_{3 \mathrm{ECG}}^{\mathrm{SR}}$, whose respiratory rate estimates obtained a very strong correlation with respect to reference, and did not show a dependence on the actual respiratory rate (see Fig. 6).

The EDR methods were modified in order to lower their computational cost, based on a decimation to $250 \mathrm{~Hz}$, a 
common QRS detection for all the ECG channels, a more simple estimation of QRS slopes (from which the R-wave angle is computed), and the combination of $d_{\mathrm{US}}^{\mathrm{LC}}(n)$ and $d_{\mathrm{DS}}^{\mathrm{LC}}(n) \mathrm{EDR}$ signals by an unconditional subtraction instead of the peakconditioned spectral average. The more simple estimation of QRS slopes consists of taking the maximum of the first derivative with no posterior line fitting. It is worthy to note that, as a decimation to $250 \mathrm{~Hz}$ was performed previously, a line fitting within $8 \mathrm{~ms}$ would take into consideration only 2 samples, leading to a slope identical to the first derivative at that instant.

Results in terms of medians and IQRs of $e(\mathrm{k})$ slightly worse in general. Furthermore, the Friedman statistical test found significant differences in $e(k)$ obtained from LC combinations with respect to their analogous combinations of the other methods: $C_{3 \mathrm{ECG}}$ and $C_{\mathrm{PCA}}$ in the case of original methods, and all three ( $C_{3 \mathrm{ECG}}, C_{\mathrm{PCA}}$, and $C_{\mathrm{ALL}}$ ) in the case of the SR methods. However, medians and IQRs of $e(k)$ were not higher than $3.57 \%$ for all stages when using $C_{\mathrm{PCA}}^{\mathrm{LC}}$, suggesting that these $\mathrm{LC}$ methods also can exploit the respiration-related modulations in armband-ECG signals to derive good estimates of the respiratory rate. $C_{\mathrm{PCA}}^{\mathrm{LC}}$ obtained the highest $\mathrm{R}_{<5 \%}$ in all six stages of the protocol, while $C_{\mathrm{ALL}}^{\mathrm{LC}}$ did in one stages, and $C_{3 \mathrm{ECG}}^{\mathrm{LC}}$ did not obtain it in any of the stages. With respect to $\mathrm{R}_{<3 \%}, C_{\mathrm{PCA}}^{\mathrm{LC}}$ obtained the highest $\mathrm{R}_{<3 \%}$ in all six stages of the protocol, while $C_{\mathrm{ALL}}^{\mathrm{LC}}$ and $C_{3 \mathrm{ECG}}^{\mathrm{LC}}$ did not obtain it in any of the stages. This suggest that in the case of LC methods, the information added by the EDR signals from $x_{\mathrm{PCA}}(n)$ complements the information of EDR signals from the original ECG channels, leading to higher number of recordings with accurate respiratory rate estimates.

The mean of computational time per minute using Matlab R2017b on an Intel Core I7 4470 was measured. These computational times should not be extrapolated to those that would be measured on wearable devices, as their microprocessors' computational speed is usually more limited. However, the comparison between them can provide an idea of the relative speed of one method with respect to the other, even if these relations may change when using a different computational microprocessor. Computational times were considerably lower for the $C_{3 \mathrm{ECG}}^{\mathrm{LC}}$ and $C_{\mathrm{ALL}}^{\mathrm{LC}}$ methods than for their corresponding original methods $(76.17 \%$ and $76.57 \%$, respectively), and a more moderate improvement (15.23\%) in these terms was observed in the case of $C_{\mathrm{PCA}}^{\mathrm{LC}}$. Again, $C_{3 \mathrm{ECG}}$ and $C_{\mathrm{ALL}}^{\mathrm{LC}}$ obtained similar results in these terms (1076.89 ms and $1088.29 \mathrm{~ms}$, respectively). The $C_{\mathrm{PCA}}^{\mathrm{LC}}$ obtained a slightly lower computational time per minute of signal (988.53 ms). Thus, our recommendation for fast estimation of respiratory rate is to use the $\mathrm{LC}$ version of $C_{\mathrm{PCA}}^{\mathrm{LC}}$, which reduced the computational time a $77.01 \%$ with respect to our recommendation for accurate respiratory rate estimation using the QRS-slopes range approach $\left(C_{3 \mathrm{ECG}}^{\mathrm{SR}}\right)$, and a $78.13 \%$ with respect to its associated version in original methods in [21] $\left(C_{3 \mathrm{ECG}}\right)$. However, this improvement of computation time comes at the cost of a decrease in accuracy of the estimation. In fact, estimations of $C_{\mathrm{PCA}}^{\mathrm{LC}}$ showed a lower correlation with the reference, although still strong (0.87). Furthermore, a slight tendency to underestimate the respiratory rate can be observed in the BlandAltman plot (see Fig. 6).

Computational time and accuracy are a trade-off. A good solution is $C_{\mathrm{PCA}}^{\mathrm{SR}}$, whose estimates did not show significant differences with those of $C_{3 E C G}^{\mathrm{SR}}$ (our recommendation for accurate estimates), while the computational time was only slightly higher $(12.45 \%)$ than $C_{\mathrm{PCA}}^{\mathrm{LC}}$ (our recommendation for fast estimates). In addition, estimations from $C_{\mathrm{PCA}}^{\mathrm{SR}}$ showed a very high correlation with the reference (0.97), and did not show a dependence on the actual respiratory rate. However, it should be kept in mind that $C_{\mathrm{PCA}}^{\mathrm{SR}}$ uses a sampling rate of 1000 $\mathrm{Hz}$ while $C_{\mathrm{PCA}}^{\mathrm{LC}}$ uses a sampling rate of $250 \mathrm{~Hz}$, with implications for battery consumption and required memory of the final device.

Those fragments associated with a respiratory rate higher than half the mean heart rate were also excluded, because the EDR methods would track an alias rate in such situations. This was the case for two subjects at the stage of controlled breathing at $0.5 \mathrm{~Hz}$. This is the consequence of forcing the subjects to breathe at a high rate while the body is not demanding it. A high respiratory rate with a low heart rate does not represent a realistic physiological situation. In such situations when the autonomic nervous system requires a high respiratory rate, it also requires a high heart rate (which leads to a high pulse rate), e.g., during exercise. However, this issue, which affects all beat-to-beat EDR methods, remains a limitation for monitoring patients with a non-physiological heart rate (such as those treated with beta-blockers).

A limitation of this study is that the respiratory rate estimation has been evaluated only with healthy subjects, as some common problems may appear in specific applications including respiratory related pathologies. Even if successfully demonstrated that the armband can accurately track respiratory rates, its behavior during pathological respiratory events such as obstructive apnea has to be examined. Further studies have to be conducted in order to evaluate the usefulness of respiratory information obtained from the armband in specific applications. However, the respiratory rate tracking in healthy subjects is useful in some applications, such as general wellbeing and stress level assessment [10].

Although there were no segments from which respiratory rate could not be estimated, during any of the studied stages and any of the studied EDR sets, this is not the normal expectation when monitoring daily life. In this study, the subjects were not moving, but EMG artifacts are certainly expected in the armband ECG signals during daily monitoring. It is noted that in [21], the peakness-based criterion showed good performance in detecting those EDR segments from which an accurate respiratory rate can be obtained. In addition, ECG signal quality indices can be used as a first step in order to detect when good quality ECG signal is available and when it is not. A recent study revealed that the armband can provide usable ECG data (for detecting QRS complexes) during daily life in more than $75 \%$ (in median) of the non-bed time and more than $98 \%$ of the bed time, while the percentages of usable data found by an automatic strict artifact detector were 53\% and 95\% for nonbed and bed time, respectively [26]. Thus, a high number of artifacts are expected during daily life, however, the armband device can be worn for long periods of time (months or even 
years), leading to a much higher total amount of quality data than the alternatives based on Holter, event, or patch monitors.

\section{CONCLUSSIONS}

Respiration-related modulation of the ECG morphology can be observed in the ECG signals recorded by the armband wearable device based on three pairs of dry electrodes worn over the upper left arm. Furthermore, this modulation can be exploited by the EDR method based on QRS slopes and R-wave angles for deriving respiratory rates with a relative error not higher than $1.40 \%$ in median (inter-subject median of median) and $3.06 \%$ in IQR (inter-subject median of IQR) when using the $\mathrm{C}_{3 \mathrm{ECG}}$ set. Computational time can be reduced a $78.13 \%$ assuming a lower accuracy, specifically, the highest median of relative error was $0.27 \%$, and its highest IQR was $3.57 \%$ when using the $C_{\mathrm{PCA}}^{\mathrm{LC}}$ set.

These results are promising and allow us to consider the armband ECG device as a potential wearable cardiorespiratory monitor which can be worn daily for long periods of time (months or years), having a wide range of potential applications including monitoring patients with chronic respiratory diseases, epileptic seizures detection, stress assessment, and sleep studies, among others.

\section{REFERENCES}

[1] B. Krieger, D. Feinerman, A. Zaron, and F. Bizousky, "Continuous noninvasive monitoring of respiratory rate in critically ill patients," Chest, vol. 90, no. 5, pp. 632-634, Nov. 1986.

[2] T. R. Gravelyn and J. G. Weg, "Respiratory rate as an indicator of acute respiratory dysfunction,” JAMA, vol. 244, no. 10, pp. 1123-1125, Sep. 1980.

[3] A. Garde et al., "Time-varying signal analysis to detect high-altitude periodic breathing in climbers ascending to extreme altitude," Med. Biol. Eng. Comput., vol. 53, no. 8, pp. 699-712, Aug. 2015, doi: 10.1007/s11517-015-1275-x.

[4] D. P. Francis, K. Willson, L. C. Davies, A. J. Coats, and M. Piepoli, "Quantitative general theory for periodic breathing in chronic heart failure and its clinical implications," Circulation, vol. 102, no. 18, pp. 2214-2221, Oct. 2000.

[5] M. A. Cretikos, R. Bellomo, K. Hillman, J. Chen, S. Finfer, and A. Flabouris, "Respiratory rate: the neglected vital sign," Med. J. Aust., vol. 188, no. 11, pp. 657-659, Jun. 2008.

[6] R. Parkes, "Rate of respiration: the forgotten vital sign," Emerg. Nurse J. RCN Accid. Emerg. Nurs. Assoc., vol. 19, no. 2, pp. 12-17; quiz 18, May 2011, doi: 10.7748/en2011.05.19.2.12.c8504.

[7] A. Nicolò, C. Massaroni, and L. Passfield, "Respiratory Frequency during Exercise: The Neglected Physiological Measure," Front. Physiol., vol. 8, Dec. 2017, doi: 10.3389/fphys.2017.00922.

[8] C. Varon, A. Caicedo, D. Testelmans, B. Buyse, and S. Van Huffel, “A Novel Algorithm for the Automatic Detection of Sleep Apnea From Single-Lead ECG," IEEE Trans. Biomed. Eng., vol. 62, no. 9, pp. 22692278, Sep. 2015, doi: 10.1109/TBME.2015.2422378.

[9] C. Varon, K. Jansen, L. Lagae, and S. Van Huffel, "Can ECG monitoring identify seizures?,” J. Electrocardiol., vol. 48, no. 6, pp. 1069-1074, Dec. 2015, doi: 10.1016/j.jelectrocard.2015.08.020.

[10] A. Hernando et al., "Inclusion of Respiratory Frequency Information in Heart Rate Variability Analysis for Stress Assessment," IEEE J. Biomed. Health Inform., vol. 20, no. 4, pp. 1016-1025, 2016, doi: 10.1109/JBHI.2016.2553578.

[11] J. Milagro et al., "Nocturnal Heart Rate Variability Spectrum Characterization in Preschool Children With Asthmatic Symptoms," IEEE J. Biomed. Health Inform., vol. 22, no. 5, pp. 1332-1340, 2018, doi: 10.1109/JBHI.2017.2775059.

[12] U. Frey and B. Suki, "Complexity of chronic asthma and chronic obstructive pulmonary disease: implications for risk assessment, and disease progression and control," Lancet Lond. Engl., vol. 372, no. 9643, pp. 1088-1099, Sep. 2008, doi: 10.1016/S0140-6736(08)61450-6.

[13] J. Vestbo et al., "Global strategy for the diagnosis, management, and prevention of chronic obstructive pulmonary disease: GOLD executive summary," Am. J. Respir. Crit. Care Med., vol. 187, no. 4, pp. 347-365, Feb. 2013, doi: 10.1164/rccm.201204-0596PP.

[14] H.-C. Jung et al., "CNT/PDMS composite flexible dry electrodes for long-term ECG monitoring," IEEE Trans. Biomed. Eng., vol. 59, no. 5, pp. 1472-1479, May 2012, doi: 10.1109/TBME.2012.2190288.

[15] Y. T. Tsukada et al., "Validation of wearable textile electrodes for ECG monitoring," Heart Vessels, Jan. 2019, doi: 10.1007/s00380-019-013478.

[16] B. A. Reyes et al., "Novel electrodes for underwater ECG monitoring," IEEE Trans. Biomed. Eng., vol. 61, no. 6, pp. 1863-1876, Jun. 2014, doi: 10.1109/TBME.2014.2309293.

[17] R. Bailón, L. Sörnmo, and P. Laguna, “A robust method for ECG-based estimation of the respiratory frequency during stress testing," IEEE Trans. Biomed. Eng., vol. 53, no. 7, pp. 1273-1285, Jul. 2006, doi: 10.1109/TBME.2006.871888.

[18] P. H. Charlton, T. Bonnici, L. Tarassenko, D. A. Clifton, R. Beale, and P. J. Watkinson, "An assessment of algorithms to estimate respiratory rate from the electrocardiogram and photoplethysmogram," Physiol. Meas., vol. 37, no. 4, pp. 610-626, Apr. 2016, doi: 10.1088/09673334/37/4/610.

[19] Y. Nam, J. Lee, and K. H. Chon, "Respiratory rate estimation from the built-in cameras of smartphones and tablets," Ann. Biomed. Eng., vol. 42, no. 4, pp. 885-898, Apr. 2014, doi: 10.1007/s10439-013-0944-x.

[20] J. A. Hirsch and B. Bishop, "Respiratory sinus arrhythmia in humans: how breathing pattern modulates heart rate,” Am. J. Physiol., vol. 241, no. 4, pp. H620-629, Oct. 1981, doi: 10.1152/ajpheart.1981.241.4.H620.

[21] J. Lázaro et al., "Electrocardiogram derived respiratory rate from QRS slopes and R-wave angle," Ann. Biomed. Eng., vol. 42, no. 10, pp. 2072-2083, Oct. 2014, doi: 10.1007/s10439-014-1073-x.

[22] J. Lázaro, R. Bailón, E. Gil, Y. Noh, P. Laguna, and K. H. Chon, "Pilot study on electrocardiogram derived respiratory rate using a wearable armband,” presented at the XLV International Conference on Computing in Cardiology, 2018.

[23] C. Varon et al., "A comparative study of ECG-derived respiration in ambulatory monitoring using the single-lead ECG," Sci. Rep., 2020.

[24] S. K. Bashar, A. J. Walkey, D. D. McManus, and K. H. Chon, "VERB: VFCDM-Based Electrocardiogram Reconstruction and Beat Detection Algorithm," IEEE Access, pp. 1-1, 2019, doi: 10.1109/ACCESS.2019.2894092.

[25] S. Kontaxis et al., "ECG-derived Respiratory Rate in Atrial Fibrillation,” IEEE Trans. Biomed. Eng.

[26] J. Lázaro, N. Reljin, M.-B. Hosain, Y. Noh, P. Laguna, and K. H. Chon, "Wearable armband device for daily life electrocardiogram monitoring," IEEE Trans. Biomed. Eng., 2020, accepted. 